\title{
SIESTA: Thrombectomy as you like
}

\author{
Martin Bendszus ${ }^{1}$
}

Published online: 25 January 2017

(c) Springer-Verlag Berlin Heidelberg 2017

Thrombectomy has become the treatment of choice for stroke due to large vessel occlusion; however, there has been a lot of debate on the mode of patient management during thrombectomy. Recent retrospective data suggested a worse outcome in patients undergoing general anesthesia when compared to conscious sedation only [1]; however, these data have been criticized due to a potentially severe selection bias. A post hoc analysis of the MR CLEAN trial also indicated a more favorable outcome in patients not undergoing general anesthesia [2] but all of these studies compared either different patients or centers. These effects may have been much stronger than the mode of patient management during the intervention; therefore, a single center randomized controlled trial Sedation vs. Intubation for Endovascular Stroke Treatment (SIESTA) was conducted to compare general anesthesia and conscious sedation in patients with large vessel occlusion [3]. All patients were treated by an experienced team of neurointerventionalists and stroke specialists, and randomization was performed prior to treatment. Primary outcome, i. e. early neurological improvement in the NIHSS after $24 \mathrm{~h}$, was not significantly different between the general anesthesia group vs. the conscious sedation group. In the general anesthesia vs. the conscious sedation group, substantial patient movement was less frequent, but postinterventional complications were more frequent for hypothermia, delayed extubation, and pneumonia. More patients were functionally independent (unadjusted mRS score $0-2$ after 3 months in

Martin Bendszus

martin.bendszus@med.uni-heidelberg.de

1 Abteilung für Neuroradiologie, Universitätsklinikum Heidelberg, Im Neuenheimer Feld 400, 69120 Heidelberg, Germany
$37.0 \%$ of the general anesthesia group vs. $18.2 \%$ of the conscious sedation group, $P=0.01)$. There were no differences in mortality at 3 months ( $24.7 \%$ in both groups). The authors concluded that the study findings do not support an advantage for the use of conscious sedation.

What consequences can be drawn for clinical practice from this trial? According to this trial both general anesthesia and conscious sedation are safe and feasible in neurothrombectomy, and there should not be an a priori preference for either technique. It should be left up to the neurointerventionalist to decide how to safely perform the procedure. On the other hand, it has to be considered that this study was conducted under optimized conditions (i. e. dedicated stroke physicians with experience in quick and effective handling of stroke patients). Under other conditions (e. g. long waiting time for anesthesiology) the results may be different. In these instances, conscious sedation may be a better choice. The results of the SIESTA trial leave it up the choice of the interventionalist for either techniques of patient management.

Conflict of interest M. Bendszus declares that he has no competing interests.

\section{References}

1. Brinjikji W, Murad MH, Rabinstein AA, Cloft HJ, Lanzino G, Kallmes DF. Conscious sedation versus general anesthesia during endovascular acute ischemic stroke treatment: a systematic review and meta-analysis. AJNR Am J Neuroradiol. 2015;36:525-9.

2. van den Berg LA, Koelman DL, Berkhemer OA, Rozeman AD, Fransen PS, Beumer D, Dippel DW, van der Lugt A, van Oostenbrugge RJ, van Zwam WH, Brouwer PA, Jenniskens S, Boiten J, Lycklama À, Nijeholt GA, Vos JA, Schonewille WJ, Majoie CB, Roos YB, MR CLEAN pretrial study group, Participating centers. Type of anesthesia and differences in clinical outcome after intraarterial treatment for ischemic stroke. Stroke. 2015;46:1257-62. 
3. Schönenberger S, Uhlmann L, Hacke W, Schieber S, Mundiyanapurath S, Purrucker JC, Nagel S, Klose C, Pfaff J, Bendszus M, Ringleb PA, Kieser M, Möhlenbruch MA, Bösel J. Effect of Conscious Sedation vs General Anesthesia on Early Neurological Improvement Among Patients With Ischemic Stroke Undergoing En- dovascular Thrombectomy: A Randomized Clinical Trial. JAMA. 2016;316:1986-96. 